\title{
Two valves in the pharynx
}

\author{
Shiroh Isono
}

Affiliation: Dept of Anesthesiology, Graduate School of Medicine, Chiba University, Chiba, Japan.

Correspondence: Shiroh Isono, Dept of Anesthesiology, Graduate School of Medicine, Chiba University, 1-8-1 Inohana-cho, Chuo-ku, Chiba, 260-8670, Japan. E-mail: shirohisonođyahoo.co.jp

@ERSpublications

The epiglottis and soft palate function as one-way valves limiting flow during inspiration and expiration http://ow.ly/85JB30epWOC

Cite this article as: Isono S. Two valves in the pharynx. Eur Respir J 2017; 50: 1701496 [https://doi.org/ 10.1183/13993003.01496-2017].

Obstructive sleep apnoea (OSA) is caused by repetitive closure of the upper airway during sleep. While the retropalatal airway is reported to be the most collapsible site [1], any state-dependent segments within the upper airway are candidates for closure. Correct identification of the closure site in each OSA patient could lead to the development of individualised OSA treatment strategies [2]. In this issue of the European Respiratory Journal, AzARBARZIN et al. [3] propose a model for prediction of epiglottic collapse for each breath by assessing the nasal airflow waveform in sleeping OSA patients. They employed a machine-learning approach to identify characteristic waveform features for constructing the predictive algorithm and validation of the final predictive model. They found that a nasal airflow signal with greater discontinuity index (rapid and marked reduction of inspiratory airflow immediately after achieving the maximum airflow) and greater jaggedness index (repeated deviations of the airflow from the mean value during inspiration) predicts epiglottic collapse. Furthermore, the non-calibrated nasal pressure signal is demonstrated to be equally usable for determining the epiglottic collapse, increasing applicability to clinical practice. However, it is noteworthy that this study does not completely clarify why the discontinuity and jaggedness features are produced by the epiglottic collapse. Whereas application of machine-learning and artificial intelligence technologies is a promising means for solving multifactorial difficult medical problems, as is proved in this study, these technologies should be utilised through careful selection of physiologically and clinically meaningful variables in order to avoid inclusion of meaningless variables in the prediction model. So, what is the physiology behind the success of the prediction?

\section{A collapsible tube as a mechanical model for the pharyngeal airway}

The pharynx is considered to behave like a collapsible tube. This analogy leads to the application of a Starling resistor model to the upper airway, whereby the critical closing pressure $\left(P_{\text {crit }}\right)$ is obtained by an empirically observed linear relationship between the maximum inspiratory airflow and upstream pressure [4]. Notably, pioneer researchers were well aware of the existence of various inspiratory airflow patterns, while only the maximum airflow values were used to obtain Pcrit. A common feature during inspiratory flow limitation is a flattened or progressively reduced inspiratory airflow signal, which is now used for automatic determination of the optimal CPAP level [5]. Behaviour of the pure Starling resistor with infinite collapsibility differs from that of the collapsible pharyngeal airway, which properties are described by a "tube law", with variable airway wall compliance [1]. Progressive retropalatal airway narrowing during inspiratory flow limitation is precisely described by Isono et al. [6]. As the flow increases, the airway pressure decreases as a consequence of kinetic energy increase of the air (Bernoulli effect) and as a result of upstream dissipative energy loss, particularly in the nasal airway. Airway pressure decrease narrows the

Received: July 232017 | Accepted after revision: Aug 072017

Conflict of interest: Disclosures can be found alongside this article at erj.ersjournals.com

Copyright @ERS 2017 
retropalatal airway in accordance with the dynamic tube law, which consequently leads to kinetic energy increase of the gas flowing through the segment. Further increase of the inspiratory effort commonly produces progressive reduction of the limited inspiratory flow (negative effort dependence) and vibration of the soft tissue (snoring). This dynamic retropalatal airway behaviour during inspiration is in good agreement with the tube law of the collapsible pharyngeal tube.

\section{The epiglottis behaves like an inspiratory valve during inspiratory flow limitation} Susceptibility to epiglottic collapse during inspiration appears to vary among OSA patients, and 20 to $30 \%$ of these patients are reported to have a collapsible epiglottis [7]. Complete epiglottic collapse appears to occur only during the inspiratory phase, as clearly observed in the sample flow tracings with normal expiratory flow and sudden reduction of inspiratory flow as evidenced by higher ratio of peak expiratory flow and inspiratory tidal volume. This sudden flow reduction could be the result of the pure Starling resistor with infinite collapsibility, and not of the collapsible tube with variable compliance. However, presence of the unstable maximum inspiratory flow at the atmospheric upstream pressure implies that the epiglottis $P_{\text {crit }}$ is below atmospheric pressure, despite the dynamic inspiratory closure, indicating behaviour contradictory to the Starling resistor model. Accordingly, neither the Starling resistor model nor the collapsible tube with variable compliance adequately describes the sudden mid-inspiratory flow reduction.

Alternatively, such a sudden change in luminal pressure can be described by a valve-like behaviour of the epiglottis. In fact, the epiglottis is structurally a peninsula in the airway space and it could function as a swing-type check valve which suddenly closes for inspiratory flow interruption and opens for normal expiration as is observed in the supplemental video of the highlighted article. Opening or closing of the swing valve is mainly determined by the pressure difference between anterior and posterior valve surfaces, valve mass and valve angle for the airflow. Application of the swing-type epiglottic valve to computational fluid dynamics would increase our understanding of the mechanisms of epiglottic collapse and inspiratory flow limitation.

\section{Discontinuity and jagged features of the inspiratory flow signal during epiglottic collapse}

The one-way valve assumption of epiglottic behaviour accounts for the higher discontinuity index for epiglottic collapse. Discontinuity of the inspiratory flow may possibly be produced by sudden epiglottic valve closure, in which timing of the closure is determined by the epiglottis mass, the airway angle at inspiratory flow initiation, and the epiglottic hinge mobility. Variability and instability of the discontinued and resumed inspiratory flow rates observed in figure 5 of the article by AzARBARzin et al. [3] suggest unstable epiglottic valve function unpredictable by the collapsible tube model.

AzArbarzin et al. [3] describe repetitive reopening/closing during inspiration producing a "jagged" flow. This jagged flow pattern is explainable by the collapsible tube model, which produces a conversion cycle of kinetic energy to potential energy at the narrow segment along the edge of the epiglottis. According to the sample tracings, the jagged flow pattern is more common when the airflow interruption is incomplete. Therefore, the jagged flow more often occurs when the valve incompletely closes the airway and cycling of the kinetic energy conversion easily develops. The cycling frequency may be influenced by the mechanical properties of the vibrating mass. When only the surface of the epiglottic mucosa vibrates, which tends to occur in concentric narrowing of the epiglottis, the frequency is similar to the soft palate vibration. However, the vibration in the sample tracings appear to be slower than the typical snoring, indicating global movement of the epiglottic body mass. This could be regarded as the chattering phenomenon typically observed as a sign of an inappropriately functioning swing-type valve, which tends to produce a large dissipative pressure on the pharyngeal mucosa and louder chattering noise.

\section{Two one-way valves in the pharynx}

There appears to be another swing-type one-way valve in the pharynx. The soft palate hangs from the hard palate like a peninsula in the airway similar to the epiglottis while the soft palate functions as an expiratory valve. In fact, both the epiglottis and soft palate function as valves for prevention of food from entering the trachea and nose, respectively during swallowing. SAFAR [8] was the first to document the possibility that expiratory obstruction during positive pressure intranasal ventilation is caused by the valve-like behaviour of the soft palate. Recently, SATO et al. [9] reported that expiratory flow limitation with a sudden sharp flow reduction immediately after peak expiratory flow, producing a greater discontinuity index, is more commonly observed in patients with severe OSA. A subsequent endoscopic study by our research group confirmed that expiratory obstruction of the soft palate causes an expiratory flow limitation in agreement with Safar's speculation (M. Okuyama and co-workers; unpublished observation). However, a similar expiratory flow limitation pattern was also reported in spontaneously breathing OSA patients, suggesting that this phenomenon is not unique to positive pressure ventilation [10]. The inspiratory 
epiglottic collapse reported by AzARBARzin et al. [3] and the retropalatal closure during expiration can be analysed and modelled using airflow dynamics of swing-type one-way valves, and are possibly determined by features representing sudden reduction of the inspiratory and expiratory flow signals. Future studies in OSA pathophysiology should consider the possible valve-like behaviour of the pharyngeal airway as well as collapsible tube-like behaviour. Establishment of a pharyngeal mechanical model closer to its anatomical structure would lead to the success in accurately and automatically determining the responsible site of OSA development and serve to accelerate the formulation of individualised OSA treatment strategies.

\section{References}

1 Isono S, Remmers JE, Tanaka A, et al. Anatomy of pharynx in patients with obstructive sleep apnea and in normal subjects. J Appl Physiol (1985) 1997; 82: 1319-1326.

2 Wellman A, Eckert DJ, Jordan AS, et al. A method for measuring and modeling the physiological traits causing obstructive sleep apnea. J Appl Physiol (1985) 2011; 110: 1627-1637.

3 Azarbarzin A, Marques M, Sands SA, et al. Predicting epiglottic collapse in patients with obstructive sleep apnoea. Eur Respir J 2017; 50: 1700345.

4 Schwartz AR, Smith PL, Wise RA, et al. Induction of upper airway occlusion in sleeping individuals with subatmospheric nasal pressure. J Appl Physiol (1985) 1988; 64: 535-542.

5 Abdenbi F, Chambille B, Escourrou P. Bench testing of auto-adjusting positive airway pressure devices. Eur Respir J 2004; 24: 649-658.

6 Isono S, Feroah TR, Hajduk EA, et al. Interaction of cross-sectional area, driving pressure, and airflow of passive velopharynx. J Appl Physiol (1985) 1997; 83: 851-859.

7 Lan MC, Liu SY, Lan MY, et al. Lateral pharyngeal wall collapse associated with hypoxemia in obstructive sleep apnea. Laryngoscope 2015; 125: 2408-2412.

8 Safar P. Ventilatory efficacy of mouth-to-mouth artificial respiration; airway obstruction during manual and mouth-to-mouth artificial respiration. J Am Med Assoc 1958; 167: 335-341.

9 Sato S, Hasegawa M, Okuyama M, et al. Mask ventilation during induction of general anesthesia: influences of obstructive sleep apnea. Anesthesiology 2017; 126: 28-38.

10 Stănescu D, Kostianev S, Sanna A, et al. Expiratory flow limitation during sleep in heavy snorers and obstructive sleep apnoea patients. Eur Respir J 1996; 9: 2116-2121. 